Article

\title{
Development of Robot-Assisted Untact Swab Sampling System for Upper Respiratory Disease
}

\author{
Joonho Seo ${ }^{1, *(1)}$, Seongbo Shim ${ }^{1}$, Haejune Park ${ }^{1}$, Junmin Baek ${ }^{1}$, Jang Ho Cho ${ }^{1}$ \\ and Nam-Hee $\mathrm{Kim}^{2, *}$ \\ 1 Medical Assistant Robot Lab., Korea Institute of Machinery and Materials, Daegu 42994, Korea; \\ sshim@kimm.re.kr (S.S.); haejune@kimm.re.kr (H.P.); jm4904@kimm.re.kr (J.B.); jangho@kimm.re.kr (J.H.C.) \\ 2 Dept. of Neurology, Dongguk University Ilsan Hospital, Goyang 10326, Korea \\ * Correspondence: jhseo@kimm.re.kr (J.S.); nheekim8@dumc.or.kr (N.-H.K.)
}

Received: 6 October 2020; Accepted: 27 October 2020; Published: 30 October 2020

\begin{abstract}
We propose a new telerobotic system for untact swab sampling to prevent the infection of medical staff during upper respiratory sample collection. The system consists of a slave robot and two master devices. The slave robot is designed to move a swab in 6 degrees of freedom within the facial area and to insert and remove the swab under remote control by an operator at the master site based on magnified imaging of the patient's facial area. The insertion and removal of the swab into and from the nostril are implemented by means of a swab insertion unit; as the counterpart to this unit, the master system also includes a swab insertion device to control the swab insertion unit remotely. A force sensor installed on the swab holder enables monitoring of the force generated when the swab touches the target. In experiments, a virtual specimen was installed on the posterior nasopharynx wall of a life-size head phantom model. The nasopharyngeal swab samplings of the phantom model were successfully performed thanks to the force monitoring capability of the proposed telerobotic system, showing that this system is suitable for remote upper respiratory sample collection.
\end{abstract}

Keywords: swab sampling; medical robotics; parallel robots; telerobotics

\section{Introduction}

Due to the global epidemic of respiratory diseases such as Coronavirus Disease 2019 (COVID-19), interest in so-called "untact technology" is on the rise. As one type of untact technology, a master-slave medical robotic system can be utilized in telemedical operations for noncontact diagnosis and treatment.

Telerobotic systems for use in the medical field have mainly been studied for application in minimally invasive surgery (MIS) and natural orifice transluminal endoscopic surgery (NOTES) [1]. In comparison to conventional laparoscopic/endoscopic surgery, these robotic systems enable more accurate and precise manipulations by scaling or filtering the operator's motions during a surgical procedure [2,3]. Telerobotic operation procedures have also been exploited to avoid the exposure of medical staff to radiation during X-ray or computed tomography (CT) imaging. In particular, studies using master-slave robotic systems have been actively conducted in the context of surgeries performed under fluoroscopic guidance, such as catheter driving for vascular interventional procedures [4,5]. A remote needle insertion robot inside a CT scanner has also been developed for spinal intervention [6]. 
Robot-assisted tele-echography is a representative class of master-slave medical robotic systems. Ultrasound (US) imaging has many advantages, such as being noninvasive and harmless to the human body and enabling immediate diagnosis. However, different medical experts are needed for each area of diagnosis, and the diagnostic results are highly dependent on the medical staff's experience and skill [7]. Robotic tele-echography systems enable US experts in urban hospitals to control the motion of US probes through robotic manipulation and diagnose patients in remote areas without the need to travel long distances [8,9].

We are currently attempting to apply a master-slave robotic system for remote sample collection for the diagnosis of respiratory disease. To date, respiratory specimen collection has been performed only offline, meaning that medical staff are required to be in close contact with the patient for such an examination. For sampling for the high infective diseases, basic personal protective equipment (PPE) including mask, goggle, full-body protective clothing, and gloves should be worn to protect the medical staff from cross-infection. However, PPE poses a number of troubles for medical staff, such as the risk of infection when replacing it for each patient, heat stress, time consuming about 20 to $30 \mathrm{~min}$ for wearing and taking off, and restricted movement, etc. Therefore, the necessity for non-face-to-face sampling is becoming urgent as these contact sampling issues and the rapid spread and highly infectious nature of respiratory diseases.

Viral transmission is known to occur through close contact and via respiratory droplets produced by people who are suspected to be infected [10]. Since coughing or sneezing of a patient during sample collection can directly result in the infection of an examiner working in front of the patient, high-level PPE (higher than Level D) is strongly recommended for protection during such a procedure. However, wearing PPE can impair visibility by face shields, reduce dexterity due to multiple thick glove layers, and cause heat stress and dehydration [11]. Moreover, in a pandemic situation where a large number of suspected patients need to be screened for infection, during frequent changing of the disposable PPE, or if PPE is lacking, medical staff may be at a greater risk of infection. Especially in the case of COVID-19, since many patients without any symptoms nevertheless have a high potential for transmission [12], the accuracy of the initial diagnosis is very important. In this regard, robot-assisted sample collection is expected to play an important role in realizing "untact" and "accurate" respiratory specimen sampling.

At present, specimen sampling from the upper respiratory tract is mainly performed through both oropharyngeal (OP) and nasopharyngeal (NP) swab sampling. In the case of OP swab sampling, samples are collected by rubbing cotton swabs several times on the posterior pharyngeal wall and palatine tonsil. In the case of NP swab sampling, the examiner inserts a flexible swab into the nostril parallel to the palate until resistance due to contact with the posterior nasopharynx wall is felt. Once the swab reaches the posterior nasopharynx wall, it must be gently rubbed against the tissue to collect sufficient samples [13].

Although robotic systems for respiratory specimen sampling have not previously been actively studied, a robotic system for OP swabbing, which was jointly developed by the Guangzhou Institute of Respiratory Health and the Shenyang Institute of Automation under the Chinese Academy of Sciences has been introduced in [14]. This robotic system consists of a snake-shaped mechanical arm with a binocular endoscope, wireless transmission equipment, and a human-computer interaction terminal. The mechanical arm can be used to collect throat swabs on the basis of high-definition 3D anatomical scenes viewed through the endoscope. 
In this paper, we focus on a master-slave robotic system for remote NP swab sampling. Specifically, an optimized design of the slave robot and master devices for remote swab sampling and experimental results obtained by using the realized robotic system are introduced. The developed robotic system for sample collection consists of slave and master subsystems. Section 2 presents the details of these subsystems. The experimental results presented in Section 3 show the tracking accuracy of the slave robot under manipulation by the master devices, the results of force sensing, and the sample collection results achieved in NP swab tests with a head phantom model.

\section{Materials and Methods}

\subsection{Slave System}

The slave system is composed of a sample collecting robot (slave robot) and a robot base. The motions necessary for NP swab insertion seem relatively simple compared to those of other surgical procedures. However, the kinematic structure should be designed to move the swab in multiple degrees of freedom so that the position and orientation of the swab can be adapted for each patient. We apply a parallel kinematic manipulator (PKM) in the slave robot. In comparison to a serial robotic arm, a PKM is generally known to have a limited workspace and complex singularity behavior; however, others of its features, such as a higher payload-to-weight ratio, greater stiffness, greater accuracy, and higher speed, are attractive for the design of a small and robust positioning mechanism $[15,16]$.

Specifically, a rotary Stewart platform is applied for the slave robot. This platform has six servo motors with forearms and rods connected by ball bearings. The six rods support a top plate, onto which a swab insertion unit is fixed. A disposable NP swab is installed in a swab holder and inserted into the swab insertion unit. The swab insertion unit then moves the swab holder back and forth with respect to the patient through the hole in the base frame. This hole is designed to be $120 \mathrm{~mm}$ in diameter to cover the human facial area for NP swab sampling. We establish an elliptic workspace with diameters of $60 \mathrm{~mm} \times 80 \mathrm{~mm}$ around both nostrils. The swab insertion unit can insert the NP swab up to a depth of $150 \mathrm{~mm}$, considering the depth of the posterior nasopharynx from the nostrils. The slave robot is also equipped with a camera with a wide-angle lens to transfer images of the patient's face to the master site in real time.

The slave robot is fixed to the robot base. The robot base is placed next to the subject such that the slave robot is located in front of the patient's face, as shown in Figure 1. To maintain distance from the slave robot to the patient's face and ensure that the face is located in the center of the slave robot, a head \& chin rest is mounted on the robot base, as shown in Figure 1a,b. The patients will voluntarily place their face to the head \& chin rest. If the patients feel inconvenience during the swab insertion, they can pull their head back.

As shown in Figure 2, the swab insertion unit of the slave robot is implemented by means of a double roller mechanism. To push and pull the swab, a double roller structure is adopted rather than a linear actuator by ball screw. This can prevent the further advance of the swab due to slippage if the swab holder impacts the target with a force greater than the friction force between the rollers and the rod of the swab holder. The friction force can be adjusted by modifying the gap between two rollers as the value of " $d$ " in Figure 2a. Currently, the swab insertion unit of the slave robot can apply a maximum force of $0.9 \mathrm{~kg} \cdot \mathrm{f}$ to the swab holder, as measured in experiments. Two servo motors face each other and rotate in the same direction at up to 106 RPM. Since the roller diameter is $22 \mathrm{~mm}$, the maximum speed of the linear motion of the swab holder is approximately $122 \mathrm{~mm} / \mathrm{s}$.

During swab insertion, whether the tip of the swab has penetrated sufficiently to touch the target area (Nasopharynx wall for NP swap sampling) is the most important criterion to determine the success or failure of sample collection. The camera on the slave robot allows the length of swab insertion to be visually checked; however, in general, the specimen collection with NP swabs is done by the swab insertion along the nasal passage to the nasopharynx until resistance is felt [17]. Therefore, not only visual monitoring of the swab displacement to estimate the insertion depth but 
also additional information such as the resistance force exerted at the tip of the swab are used to confirm whether it is touching the target area. The NP swab in the slave robot is a disposable medical device (Class I) clinically verified as safe, highly flexible, so easily bend when the tip touches an object. Therefore, a highly sensitive force sensor is required to measure the force generated when the swab touches the posterior nasopharynx wall. In the slave system, single-element capacitive force sensor (S8-1N, Medical Tactile Inc.) is selected because the sensor is very small and thin ( $8 \mathrm{~mm}$ in diameter with a $0.35 \mathrm{~mm}$ thickness) and also sensitive enough to measure the force when the NP swab touches an object and bends (vertical force sensing range: from $0.2 \mathrm{~g}$ to $100 \mathrm{~g}$ ). The sensor is installed between the swab holding part and swab holder rod, as shown in the 3D design illustration presented in Figure $3 \mathrm{~b}$. The disposable swab is tightly fit into the swab holding part and is replaced for each inspection.

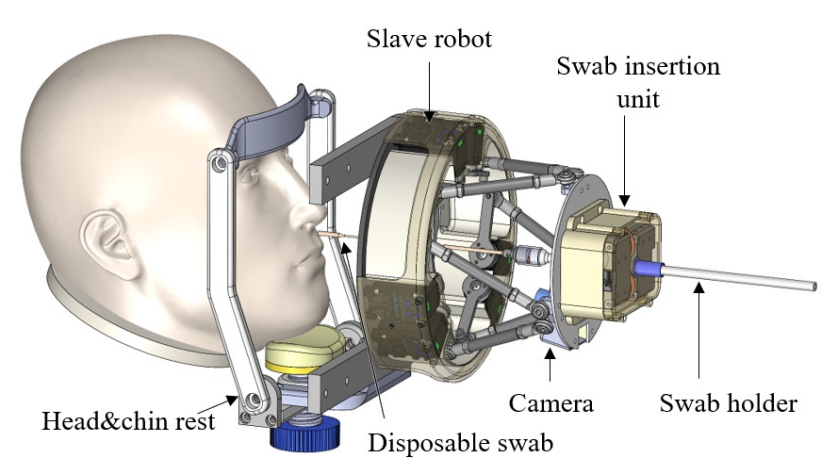

(a)

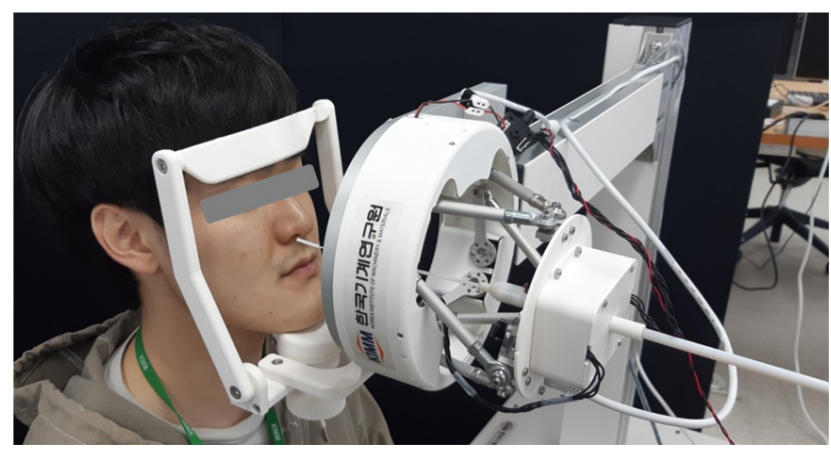

(b)

Figure 1. (a) 3D design and (b) conceptual image applying the developed prototype of the slave robot to the patient.

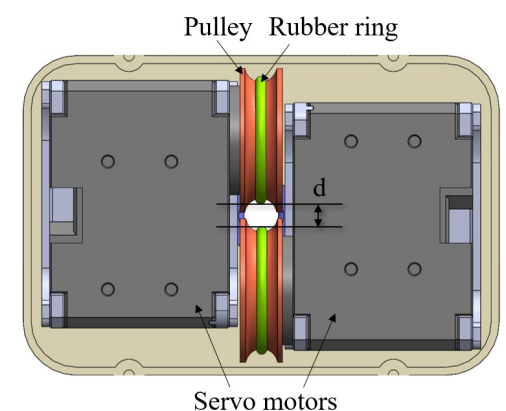

(a)

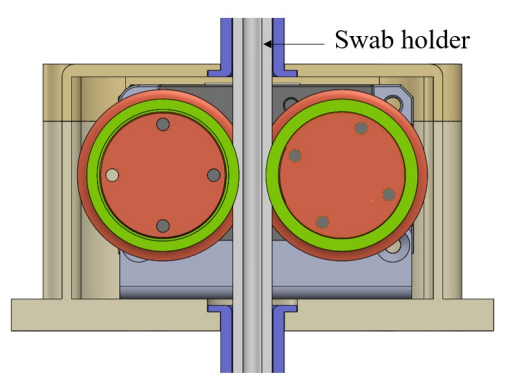

(b)

Figure 2. Mechanism of the swab insertion unit: (a) X-Z view and (b) X-Y view. 


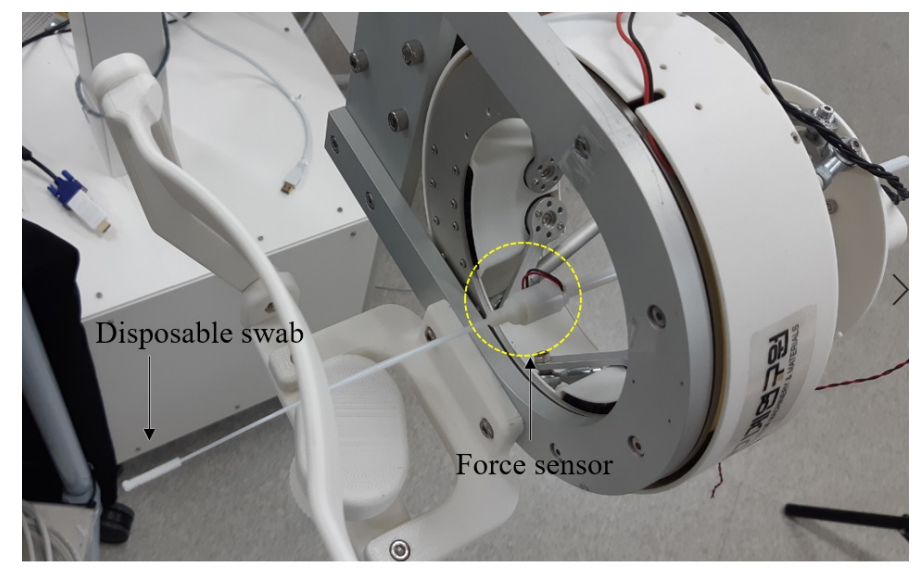

(a)

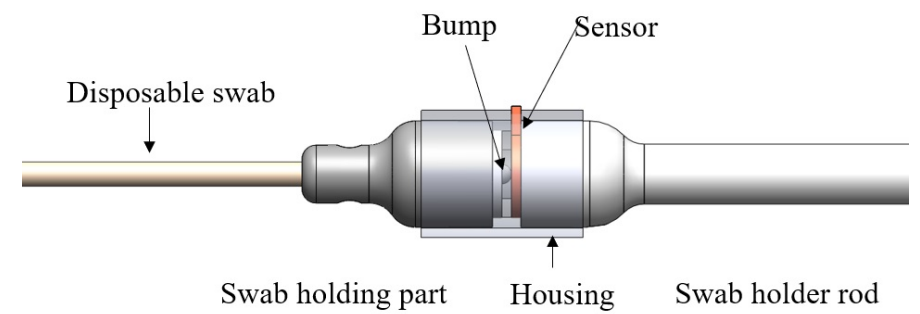

(b)

Figure 3. (a) Swab holder of the swab insertion unit of the slave robot and (b) 3D design of the force sensing part of the swab holder.

\subsection{Master System}

The master system is composed of a positioning device and a swab insertion device. The kinematic structure of the master positioning device is the same as that of the slave robot. Instead of the six motors in the slave robot, six rotary encoders record the operator's motions for swab insertion. Each encoder in the master positioning device is paired with a motor in the slave robot, and the angles recorded by the encoders are transferred to the slave site to rotate the servo motors in the same manner as the master device. This kinematically correspondent master-slave design gives the operator the ability to directly sense and control the kinematic arrangement of the slave robot arms [18]. Moreover, it allows intuitive and robust teleoperation without the need to solve for the inverse kinematics of the slave manipulator [19]. Torsion springs are mounted on the rotational shafts of the encoders for static balance compensation for the mass of the handle and for returning the assembly to the original position after manipulation. The operator at the master site holds the positioning knob as shown in Figure 4a and moves it to control the position of the swab.

Once the position and orientation of the swab have been determined, the operator can insert the swab into the nostril using the swab insertion device. The swab insertion device is designed based on a rack-and-pinion structure; pushing the swab insertion knob rotates the encoder, and the recorded length of insertion is transferred to the slave robot to move the swab insertion unit a distance equal to the received distance in real time. The swab holder of the slave robot can be moved either more slowly than or at the same rate as the master unit by means of an adjustable scale factor. During the insertion of the swab, the position and orientation can also be corrected with the positioning device to some extent. Figure $4 \mathrm{~b}$ shows the actual prototypes of the master devices. Figure 5 shows the response of the swab insertion unit when the user moves the swab insertion device from the initial position (Figure 5a) to the end position (Figure 5b). 

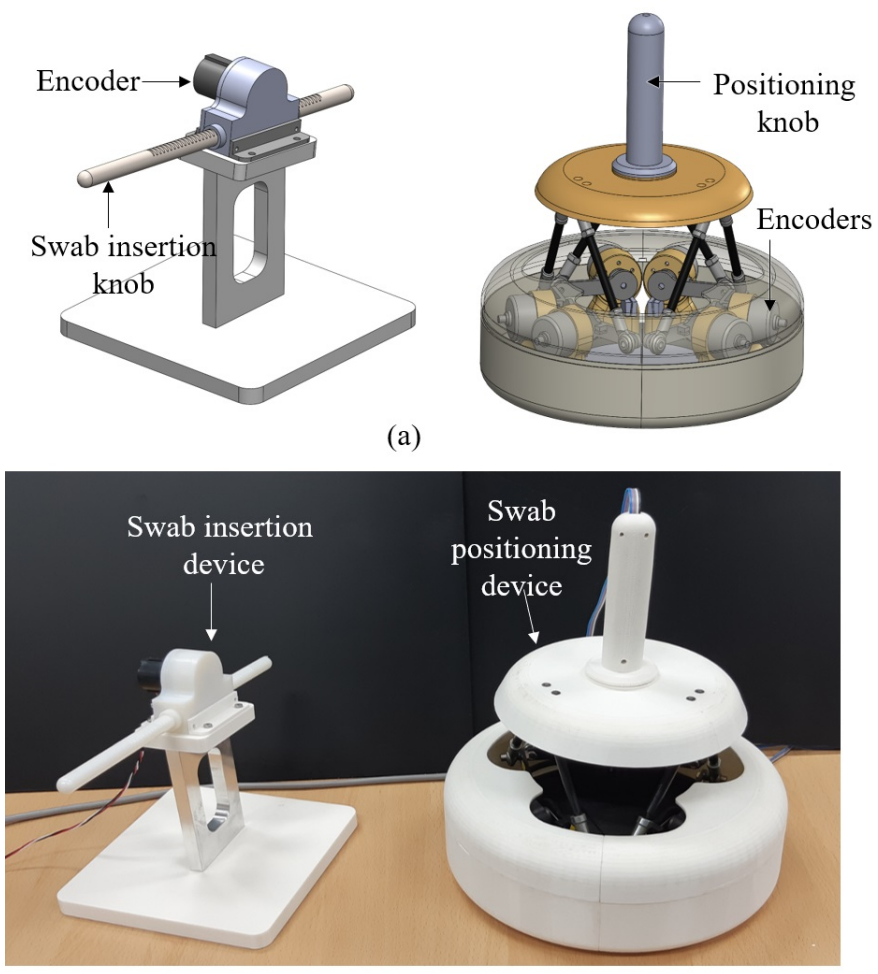

(b)

Figure 4. (a) 3D models of the swab insertion device and positioning device of the master system and (b) prototypes of the master devices.

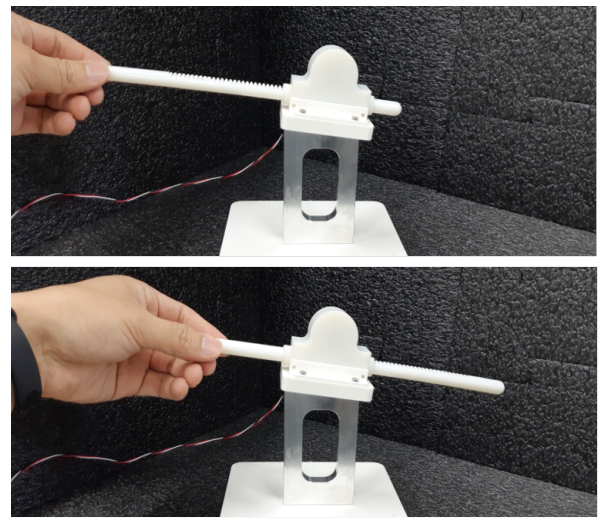

(a)
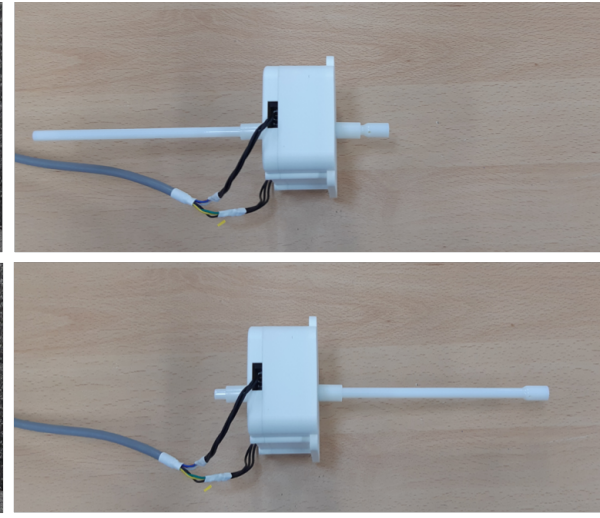

(b)

Figure 5. Test of remote swab insertion: (a) insertion motion of the swab insertion device at the master site and (b) the corresponding motion of the swab insertion unit in the slave system.

Patient monitoring software for the master site has also been developed, as shown in Figure 6. The software shows a magnified view of the patient's face as captured by the camera on the slave robot, and images of the slave and master sites are monitored as well. Additionally, the force data captured by the sensor on the swab holder of the slave robot are plotted at the bottom of the software window. All information visualized by the software is updated at $30 \mathrm{~Hz}$. 


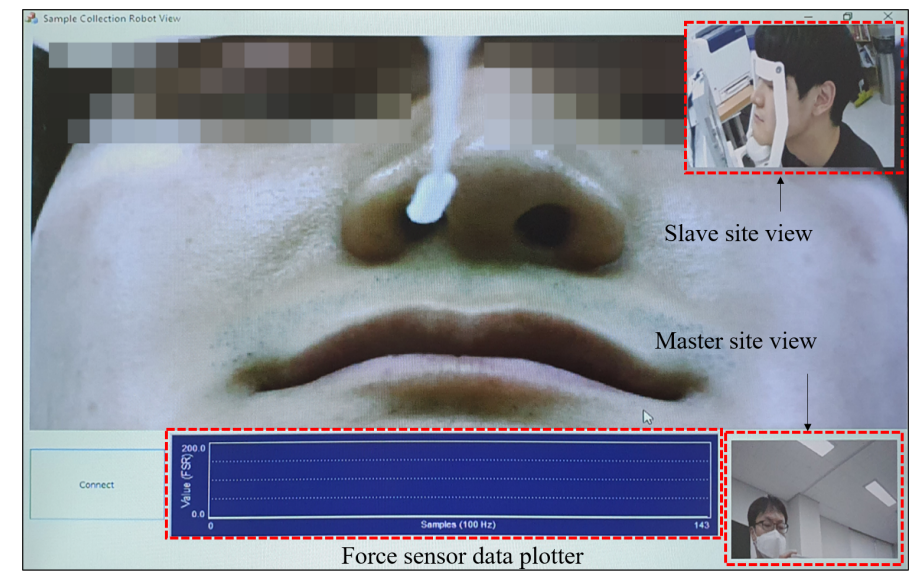

Figure 6. Screen capture of patient monitoring software viewed at the master site. A magnified facial view of the subject is displayed along with a slave site view, a master site view, and plotted force sensor data.

\subsection{Networking System}

According to the classification of telerobotic systems defined by [19], the proposed robotic system for respiratory sample collection is a "short-distance telerobotic system". Although the exact definition of a short-distance telerobotic system requires that the slave system be in the same room as the master system, for the remote sample collection procedure, it is assumed that the patient and the master operator are separated by a wall in the same hospital area. Therefore, the master and slave systems are connected via a local area network (LAN) protocol. For use in this system, a pair of hardware codecs for teleconferencing (WEB-100, Wooksung Media Inc., Daejeon, Korea) was modified to connect the master and slave sites. The hardware system transfers the robot control data, force sensor data, and facial images of the patient. Using the basic features of the teleconference system, audiovisual communication between the master and slave sites is also available. Such communication is necessary to monitor the remote environment and communicate with the patient and on-site medical staff. A camera with a microphone is located at both the slave and master sites, and the captured video and audio streams are encoded using the H.264 codec at a resolution of $1920 \times 1080$ pixels and are bilaterally communicated at 30 FPS. The video stream of the patient's face is transferred at $30 \mathrm{FPS}$ and has a resolution of $1024 \times 768$ pixels. The force sensor data sent from the slave site to the master site and the robot control data sent from the master site to the slave site are also currently transferred at $30 \mathrm{~Hz}$. Although the control frequency of $30 \mathrm{~Hz}$ is difficult to implement force feedback for a haptic master interface, this speed is sufficient for visual feedback while the examiner operates the master device [20], i.e., if the patient feels uncomfortable or the force on the swab detected by the force sensor is excessive, the examiner can recognize this through the master software in real time and withdraw the swab from the patient as soon as possible.

\section{Experimental Results}

\section{1. $2 d$ Workspace Test for Swab Positioning by the Slave Robot}

As shown in Figure 7a, the two-dimensional workspace for the NP swab is defined to cover a range of $60 \mathrm{~mm}$ in the horizontal (X-axis) and $80 \mathrm{~mm}$ in the vertical direction ( $Y$-axis), centered on the lower facial area. For patients with differently sized faces, the center of the workspace can be changed by adjusting the height of the chin rest shown in Figure 1. In these experiments, we placed a piece of gridded paper in front of the slave robot to check whether the robotic end effector could translate the swab to the borders of the workspace. The results, as presented in Figure $7 \mathrm{~b}$, show that the robotic end effector can indeed move the swab from the center of the grid (which has a scale of $10 \mathrm{~mm}$ ) to positions of $\pm 30 \mathrm{~mm}$ on the $X$-axis and $\pm 40 \mathrm{~mm}$ on the $Y$-axis. 
The length of the swab holder was also chosen by considering the typical depth to the target area. Based on the life-size phantom model used in the experiments, the depth from the nostril to the nasopharynx wall is approximately $100 \mathrm{~mm}$, as shown in Figure 7c; therefore, the swab insertion unit is designed to be able to extend the swab holder up to a depth of $150 \mathrm{~mm}$, as shown in Figure $7 \mathrm{~d}$.

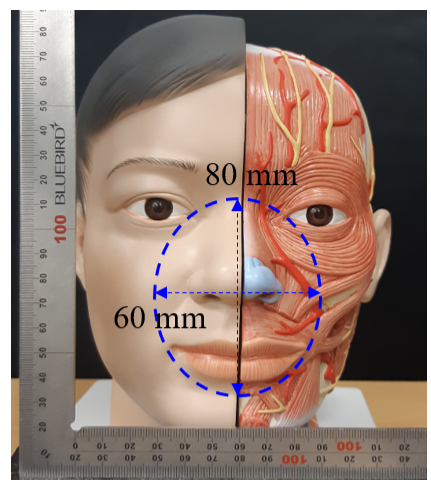

(a)

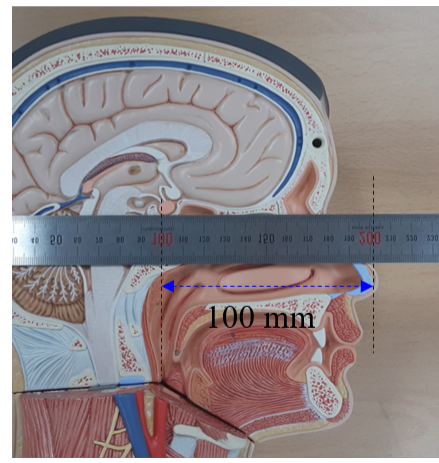

(c)

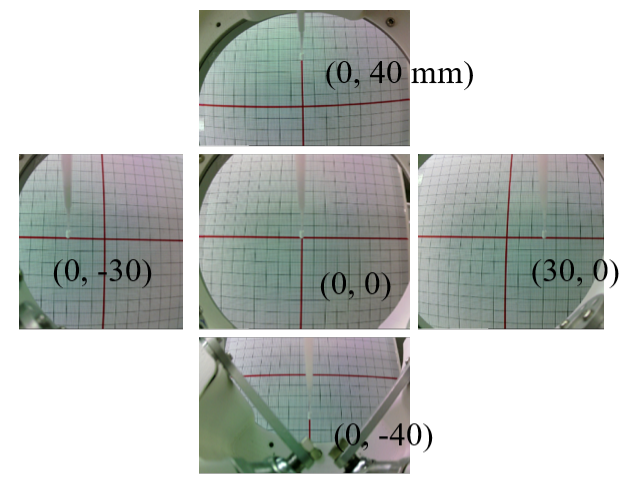

(b)

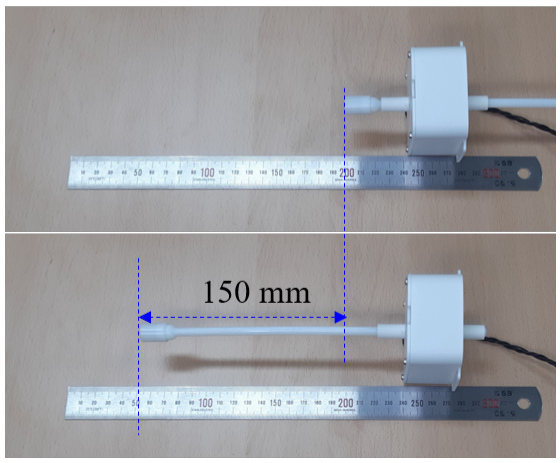

(d)

Figure 7. (a) Definition of the workspace with respect to a life-size head phantom model, (b) swab positioning test relative to the borders of the workspace, (c) depth from the nostrils to the nasopharynx wall, and (d) depth range of swab insertion.

\subsection{Motion Tracking Accuracy of the Slave Robot}

The accuracy of the slave robot's response to the motion of the master manipulator is an important performance indicator to ensure the control transparency of the telerobotic system. As shown in Figure 8a, infrared (IR) retroreflective markers were attached to the top plate of the slave robot and the end of the control knob of the master positioning device. The trajectories of both markers were recorded at $30 \mathrm{~Hz}$ by a 3D position tracker (ftk-500, Atracsys LLC, Puidoux, Switzerland). Although the kinematic structures of the slave robot and the master positioning device are identical, the positions of the markers on the slave robot and master device were different in these experiments, as shown in Figure 8a. Therefore, we transformed the measurement frame (position and orientation) of the master device into a frame identical to that of the slave robot. The tracking accuracy for each axis was defined as the average root mean square error (RMSE) of the difference between the motion trajectories of the master device and the robotic end effector in XYZ Cartesian coordinates. For example, error for $X$-axis is defined as,

$$
\epsilon_{x}=\frac{1}{M} \sum^{M} \sqrt{\frac{1}{N} \sum^{N}\left(x-x_{0}\right)^{2}}
$$


Measurements with a duration of $60 \mathrm{~s}$ were conducted 10 times. Therefore, in the expressions above, $\mathrm{N}$ is 1800, and $\mathrm{M}$ is 10 . The average error on each axis is listed in Table 1. $\epsilon_{x y z}$ is an error in 3D space calculated from $\epsilon_{x}, \epsilon_{y}$, and $\epsilon_{z}$, defined as $\epsilon_{x y z}=\sqrt{\epsilon_{x}^{2}+\epsilon_{y}^{2}+\epsilon_{z}^{2}}$. Figure $8 \mathrm{~b}$ shows one set of position tracking results between the master and slave devices on the $X_{-}, Y_{-}$, and $Z$-axes over time.

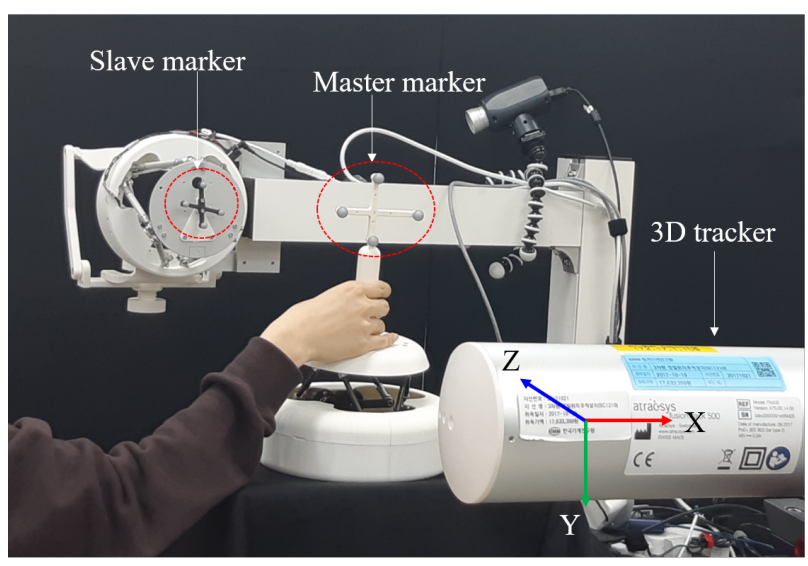

(a)

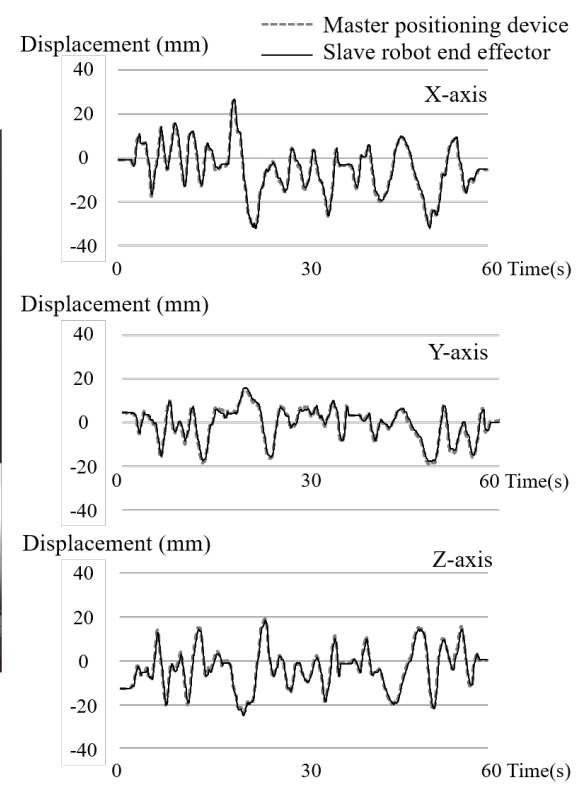

(b)

Figure 8. (a) Experimental setup for assessing the tracking accuracy of the slave robot under master manipulation, (b) X, Y, and Z motions of the slave robot (solid lines) under motion manipulation by the master positioning device (dotted line).

Table 1. Tracking errors between the positioning device of the master system and the robotic end effector in the slave system.

\begin{tabular}{cccc}
\hline$\epsilon_{x}$ & $\epsilon_{y}$ & $\epsilon_{z}$ & $\epsilon_{x y z}$ \\
\hline $1.67 \mathrm{~mm}$ & $1.26 \mathrm{~mm}$ & $2.43 \mathrm{~mm}$ & $3.41 \mathrm{~mm}$ \\
\hline
\end{tabular}

\subsection{Force Sensing of the Swab Insertion Unit}

To check the sensitivity of the force sensor mounted on the swab holder, a solid wall was installed in front of the slave robot, which was pushed toward this wall until the swab bent. As shown in Figure 9, this experiment was repeated three times, and the variation in force with the swab insertion displacement was recorded. The force sensor installed in the currently developed system does not measure the exact amount of pressing force. Instead, the magnitude of the force is scaled to a scalar value from 0 to 500. The change in the force value can also be used to estimate whether the inserted swab has reached the posterior nasopharynx wall or has touched another anatomical structure in the passage of the nasal cavity. As described in the following section, we further tested the force sensor during sample collection from a soft target installed in a head phantom. 


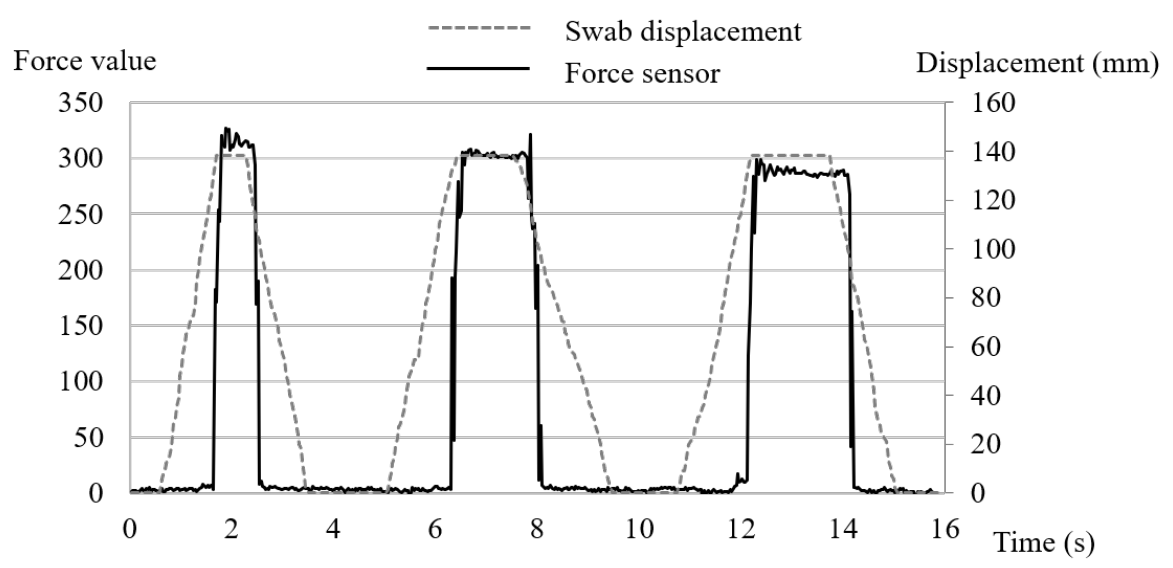

Figure 9. Variation in force with swab displacement when pressing against a solid block.

\subsection{Swab Insertion Tests Using a Head Phantom Model}

A head phantom model (Head with Neck, 3B Scientific Inc., Hamburg, Germany) was used in NP swab insertion tests. This head phantom model is a life-size model with nostrils, the nasal cavity, and bony structures in the nasopharyngeal area. In these NP swabbing experiments, a disposable swab of the same kind used for real inspections was installed in the slave robot. As shown in Figure 10, the slave robot was fixed in the robot base, which is designed for respiratory sample collection from a patient seated next to the slave robot. At the master site, the operator visually controlled the slave robot by looking at the phantom's nostrils and the swab. Since the camera and the swab are fixed on the top plate and move together with respect to the head phantom model, it is straightforward to determine the swab insertion point even from the two-dimensional images captured by a single camera.

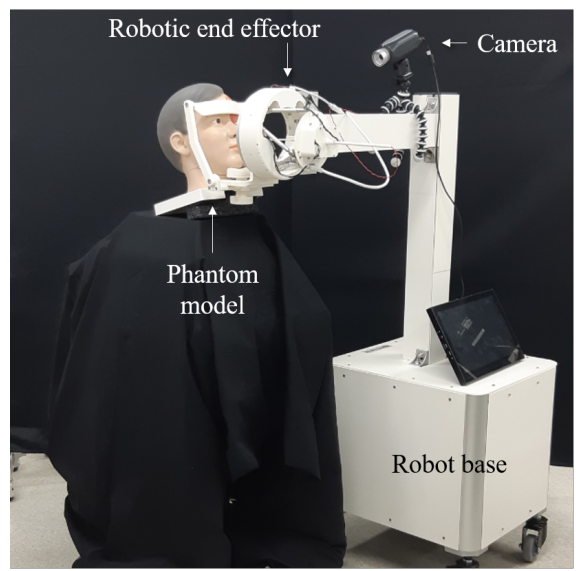

(a)

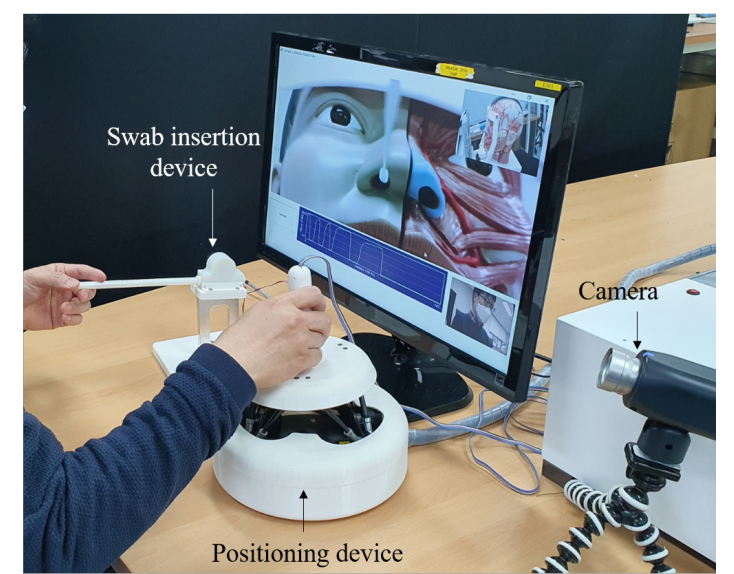

(b)

Figure 10. (a) The experimental environments of the slave site and (b) master site in the tests using the head phantom model.

Since the head phantom mode is split at the sagittal plane, it was possible to utilize specific elements of the cross-sectional anatomical structure of the head and face for testing. To test remote NP swab sampling, a virtual specimen (a cotton ball soaked with orange ink) was installed on the posterior nasopharynx wall of one section of the phantom model, as shown in Figure 11a. Visual telemanipulation of the slave robot based on facial images of the phantom model was performed as shown in Figure 10b. Figure 11b shows a clean NP swab just before insertion into the nostril, Figure 11c shows the NP swab inserted into the nostril, and Figure 11d shows the swab after extraction; the ink-smeared tip of the swab reveals that the swab successfully touched the cotton ball on the posterior nasopharynx wall. 


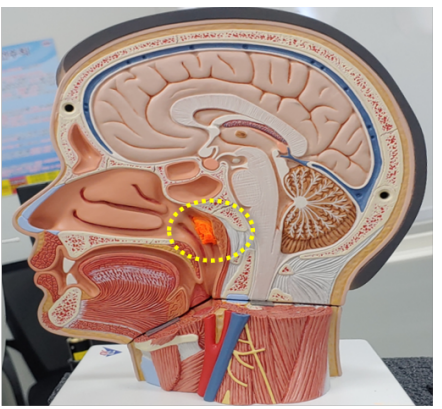

(a)

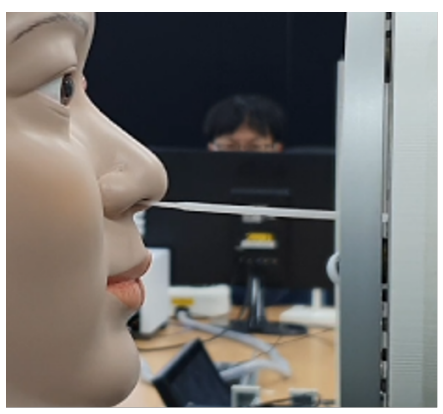

(c)

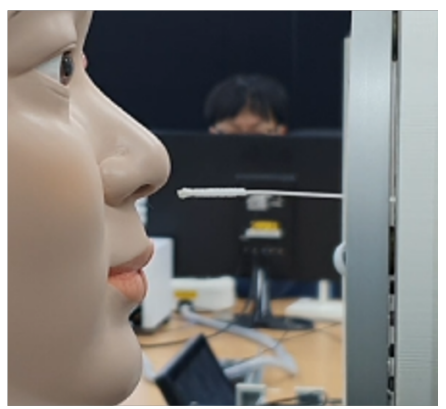

(b)

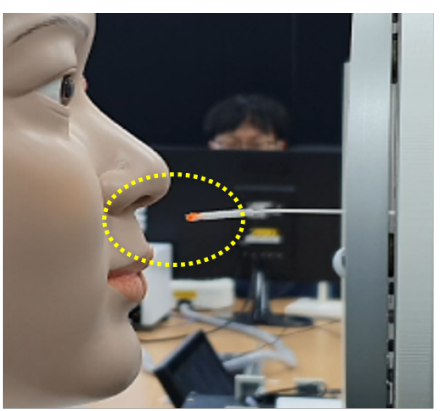

(d)

Figure 11. (a) An experiment involving swab insertion and sample collection using the head phantom model: installation of a virtual specimen (cotton ball) on the posterior nasopharynx wall, (b) before swab insertion, (c) swab insertion, and (d) swab removal after collection of the virtual sample.

During NP swab sampling, the force information was also recorded. Figure 12 shows the recorded swab displacement and force data over time. The swab was pressed against the cotton ball in the head phantom twice. If the swab is inserted in the wrong direction and touches another hard structure, as shown in Figure 9, a larger force value (300) will be recorded. However, when it touches a soft structure such as a cotton ball, a smaller force value (100) will be recorded, as shown in Figure 12. We tested NP sampling several times, and all instances of swab sampling from the cotton ball based on monitoring the change in the force data were successful. As seen from these experiments, the force data, which depend on the hardness of the anatomical structures encountered when inserting an NP swab, can be used as an important basis to determine whether sampling is performed successfully in the case of a real person.

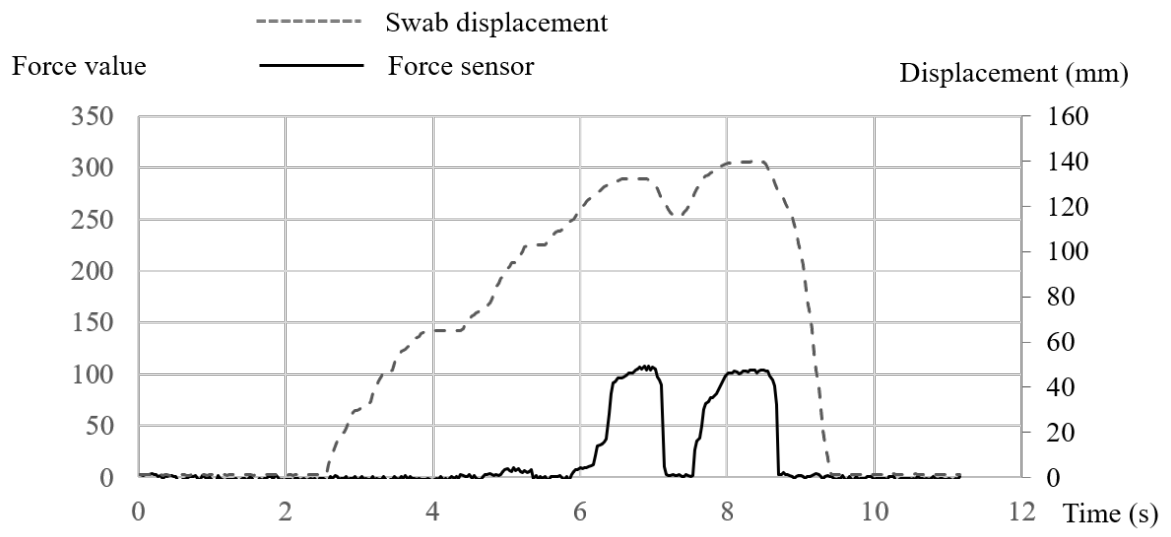

Figure 12. Experimental results of force sensing and swab displacement during nasopharyngeal (NP) swab sampling in the head phantom of Figure 11. 


\section{Discussion}

The main advantage of telerobotic sample collection is eliminating the risk of the infection of medical personnel that may occur during the collection process. However, the proposed robotic sample collection system does not support a fully "untact" sample collection process. Since the developed system allows only the process of swab sampling itself to be performed remotely, a paramedic staff member is required near the slave system to place the swab in the holder, disinfect the robot after sample collection, and reinstall a new swab. To build a fully automated sample collection system, other teleoperated systems, such as a telenursing robot [21], should be incorporated.

The proposed slave robot is designed to cover a subject's facial area; the size and kinematic structure of the slave robot are optimized for swab sampling from the upper respiratory tract while maintaining dexterity and accuracy in the facial workspace. In particular, the slave robot is designed not to be a burden to patients compared with systems that use an industrial serial robot arm, which can require large joint movements to determine the position of the swab and generate the swab insertion motion. To achieve this compact structure, however, the systems at both the master and slave sites are separated into two devices, one for position and one for insertion of swab. In the slave robot, the lengths of all six legs must be longer to cover a greater depth of swab insertion. By mounting the swab insertion unit on a Stewart platform, the insufficient Z-directional workspace of the parallel robot is complemented while maintaining the advantages of parallel robotics. For the master subsystem, two master devices have been developed for each slave unit to simplify the control method through the correspondent matching of the kinematic structures. However, in the future, these master devices will be integrated into a single unit for more intuitive operation.

In experiments, we checked whether the developed slave robot can cover the required facial workspace for NP swabbing. Additionally, experiments concerning the motion tracking accuracy of telerobotic control were conducted, and we found that the developed slave robot can track the motion of the master device with an error of $3.41 \mathrm{~mm}$ (RMSE). A life-size head phantom model was then used to test the success of sample collection using NP swabs; a virtual specimen (a cotton ball soaked with orange ink) was installed on the posterior nasopharynx wall of the model, and we tested whether our system could effectively sample the ink. By monitoring the force information sensed by the swab holder, we could recognize when the tip of the swab was pressing onto the soft cotton ball. The experimental results show that NP swabbing of the phantom could be performed successfully, thus also showing the possibility that the proposed telerobotic system could be used as an effective and safe remote sample collection system.

Since the basic feature of telerobotics is telepresence, the developed system also includes teleconference codecs that enable audiovisual communication between the slave and master sites. Data for patient imaging, robot control, and sensor monitoring are also transmitted through the teleconference system. However, due to the hardware limitations of the currently implemented teleconference system, the data transmission speed between the slave and master sites is limited to $30 \mathrm{~Hz}$, i.e., $33 \mathrm{~ms}$ of delay exists between the master device and the slave robot. These teleconference codecs will be upgraded for telerobotics, reducing the time delay between the two sites, to enable faster and more accurate visual feedback control. In addition, we plan to implement a force feedback capability to allow the master operator to feel the force generated when the swab contacts an anatomical structure during insertion. Since the minimum speed of a closed loop for force feedback is known to be $100 \mathrm{~Hz}$ [18], the current control frequency of $30 \mathrm{~Hz}$ between the master and slave systems is insufficient for this purpose.

The force sensor in the swab holder is intended to measure the force generated when the swab comes in contact with an obstacle ahead; however, it is currently used to determine the presence and softness of an obstacle rather than to measure a quantitative amount of force. To increase the accuracy of sample collection, the force data generated while in contact with the posterior nasopharynx wall or other nasal structures during NP swab sampling should be further studied through clinical trials. 
The developed system was designed for NP swab sampling. OP swab samples are less sensitive than NP samples for a variety of viruses; however, the sensitivity can be greatly improved by combining both modes of sampling [22,23]. Therefore, we plan to extend our system to OP swabs as well. In the case of OP swabbing, there are several obstacles that the swab must avoid, such as the teeth, gums, and uvula. However, unlike in NP swabbing, the target areas (tonsil or posterior wall of the pharynx) are easily visible to a facial camera in the slave robot; consequently, the procedure for OP swab might be much less challenging than NP swab sampling.

In this paper, the developed system was tested only for a phantom model with a similar anatomical structure to the real person. However, the performance of the system should be proven by clinical trials for the various cases of subjects. Through the clinical trials, a more convenient user interface to reduce training time for remote robot control can be derived as well as the robot design can be improved to overcome the interpatient difference in anatomical structure based on race, age, and gender. However, in this paper, we focused on a new mechanical structure, design, and integration of a telerobotic system optimized to the remote swab sampling of upper respiratory disease.

\section{Conclusions}

In this paper, we have proposed a new telerobotic system to prevent the infection of medical staff during upper respiratory sample collection. The proposed robotic system is composed of a slave robot and two master devices. Specifically, an optimized slave robot structure for upper respiratory sample collection is proposed. The kinematic structure is based on a rotary Stewart platform to allow the swab to be moved in 6 degrees of freedom within the facial area. The top plate of the slave robot supports the swab insertion unit, and the base hole of the slave robot provides a window for the insertion of the NP swab into the nostril of the subject. The slave robot is equipped with a wide-angle camera for the magnified imaging of the patient's facial area and transferred as an image stream to the master site. The operator at the master site controls the slave robot and visually determines the position and orientation for swab insertion by looking at the transferred images of the patient's face.

The kinematic structure of the master device is identical to that of the slave robot (a rotary Stewart platform), allowing the master operator to intuitively control the motion of the slave robot. The insertion and removal of the swab into and from the nostril are implemented by means of a swab insertion unit, which rotates a pair of rollers to push and pull the swab holder. As the counterpart to this unit, the master system includes a swab insertion device to control the swab insertion unit remotely; thus, the master operator can move the swab holder of the slave robot at the same speed desired for swab insertion. The swab holder also has a force sensor to sense the force generated when the swab touches the target. This allows the force measured during swab insertion to be monitored at the master site.

The developed system was not validated through clinical trials yet. However, the applicability of the proposed system was proved by the experiments of workspace, tracking accuracy, and virtual target sampling in the posterior nasopharynx wall of head phantom model. The experimental results also show that the proposed system is suitable to realize "untact" swab sampling for upper respiratory disease and that it could probably reduce the risk of infection and fatigue of medical staff in the event of mass infection.

Author Contributions: J.S. initiated the study, designed and produced the system, and wrote the manuscript, N.-H.K. is a coinitiator of this study and gave important medical and clinical advice and revised the manuscript, S.S. helped write manuscript and assisted experiments with J.B. and H.P. J.H.C. helped design the master manipulator and analyze the experimental results. All authors have read and agreed to the published version of the manuscript.

Funding: This work was supported by the institutional research project of Korea Institute of Machinery and Materials (No. NK226D-KIMM) and the National Research Council of Science \& Technology (NST) grant by the Korea government (MSIT) (No. CAP-18-01-KIST).

Conflicts of Interest: The authors declare no conflict of interest. 


\section{References}

1. Dogangil, G.; Davies, B.L.; Rodriguez, Y.; Baena, F. A review of medical robotics for minimally invasive soft tissue surgery. J. Eng. Med. 2010, 224, 653-679. [CrossRef] [PubMed]

2. Leal Ghezzi, T.; Campos Corleta, O. 30 Years of Robotic Surgery. World J. Surg. 2016, 40, 2550-2557. [CrossRef] [PubMed]

3. Peters, B.S.; Armijo, P.R.; Krause, C.; Choudhury, S.A.; Oleynikov, D. Review of emerging surgical robotic technology. Surg. Endosc. 2018, 32, 1636-1655. [CrossRef] [PubMed]

4. Xiang, Y.; Shen, H.; Xie, L.; Wang, H. Master-Slave Guidewire and Catheter Robotic System for Cardiovascular Intervention. In Proceedings of the 28th IEEE International Conference on Robot and Human Interactive Communication (RO-MAN), New Delhi, India, 14-18 October 2019.

5. Guo, J.; Qi, L.; Guo, S.; Meng, C.; Zhan, Q. Study on Tracking Stability for a Master-Slave Vascular Interventional Robotic System. In Proceedings of the 2019 IEEE International Conference on Mechatronics and Automation (ICMA), Tianjin, China, 4-7 August 2019; pp. 1387-1392.

6. Ju, H.; Zhang, J.; An, G.; Pei, X.; Xing, G. A robot-assisted system for minimally invasive spine surgery of percutaneous vertebroplasty based on CT images. In Proceedings of the 2008 IEEE Conference on Robotics, Automation and Mechatronics, Chengdu, China, 21-24 September 2008; pp. 290-295.

7. Arbeille, P.; Zuj, K.; Saccomandi, A.; Ruiz, J.; Andre, E.; De La Porte, C.; Carles, G.; Blouin, J.; Georgescu, M. Teleoperated Echograph and Probe Transducer for Remote Ultrasound Investigation on Isolated Patients (Study of 100 Cases). Telemed. e-Health 2016, 22, 599-607. [CrossRef] [PubMed]

8. Arbeille, P.; Zuj, K.; Saccomandi, A.; Andre, E.; De La Porte, C.; Georgescu, M. Tele-Operated Echography and Remote Guidance for Performing Tele-Echography on Geographically Isolated Patients. J. Clin. Med. 2016, 5, 58. [CrossRef] [PubMed]

9. Vieyres, P.; Poisson, G.; Courrèges, F.; Mérigeaux, O.; Arbeille, P. The TERESA project: From space research to ground tele-echography. Ind. Rob. 2003, 30, 77-82. [CrossRef] [PubMed]

10. World Health Organization. Q\&A on Coronaviruses. Available online: https://www.who.int/news-room/ q-a-detail/ q-a-coronaviruses (accessed on 6 June 2020).

11. Loibner, M.; Hagauer, S.; Schwantzer, G.; Berghold, A.; Zatloukal, K. Limiting factors for wearing personal protective equipment (PPE) in a health care environment evaluated in a randomised study. PLoS ONE 2019, 14, e0210775. [CrossRef] [PubMed]

12. Centers for Disease Control and Prevention Clinical Questions about COVID-19: Questions and Answers. Available online: https:/ / www.cdc.gov/coronavirus/2019-ncov/hcp/faq.html (accessed on 5 September 2020).

13. Centers for Disease Control and Prevention Interim Guidelines for Collecting, Handling, and Testing Clinical Specimens from Persons for Coronavirus Disease 2019 (COVID-19). Available online: https:/ /www.cdc. gov / coronavirus /2019-ncov/lab / guidelines-clinical-specimens.html (accessed on 8 September 2020).

14. Li, S.Q.; Guo, W.L.; Liu, H.; Wang, T.; Zhou, Y.Y.; Yu, T.; Wang, C.Y.; Yang, Y.M.; Zhong, N.S.; Zhang, N.F.; et al. Clinical application of an intelligent oropharyngeal swab robot: Implication for the COVID-19 pandemic. Eur. Respir. J. 2020, 56, 2001912. [CrossRef] [PubMed]

15. Merlet, J.P. Parallel Robots, 2nd ed.; Solid Mechanics and Its Applications; Springer: Dordrecht, The Netherlands, 2006.

16. Park, F.C. Parallel Robots. Encycl. Syst. Control 2015, 1031-1036. [CrossRef]

17. Marty, F.M.; Chen, K.; Verrill, K.A. How to Obtain a Nasopharyngeal Swab Specimen. N. Engl. J. Med. 2020, 382 , e76. [CrossRef] [PubMed]

18. Fischer, P.; Daniel, R.; Siva, K.V. Specification and design of input devices for teleoperation. In Proceedings of the IEEE International Conference on Robotics and Automation, Cincinnati, OH, USA, 13-18 May 1990; Volume 1, pp. 540-545.

19. Avgousti, S.; Christoforou, E.G.; Panayides, A.S.; Voskarides, S.; Novales, C.; Nouaille, L.; Pattichis, C.S.; Vieyres, P. Medical telerobotic systems: Current status and future trends. Biomed. Eng. Online 2016, 15, 1-44. [CrossRef] [PubMed] 
20. Barth, M.; Burkert, T.; Eberst, C.; Stöffler, O.; Fäerber, G. Photo-realistic scene prediction of partially unknown environments for the compensation of time delays in telepresence applications. In Proceedings of the 2000 ICRA. Millennium Conference. IEEE International Conference on Robotics and Automation. Symposia Proceedings (Cat. No.00CH37065), San Francisco, CA, USA, 24-28 April 2000; IEEE: Piscataway, NJ, USA, 2002; Volume 4, pp. 3132-3137.

21. Hauser, K.; Shaw, R. How Medical Robots Will Help Treat Patients in Future Outbreaks. IEEE Spectrum 2020. Available online: https:/ / spectrum.iee.org/automaton/robotics/medical-robots/medical-robots-futureoutbreak-response (accessed on 4 June 2020).

22. Kim, C.; Ahmed, J.A.; Eidex, R.B.; Nyoka, R.; Waiboci, L.W.; Erdman, D.; Tepo, A.; Mahamud, A.S.; Kabura, W.; Nguhi, M. Comparison of Nasopharyngeal and Oropharyngeal swabs for the diagnosis of eight respiratory viruses by real-time reverse transcription-PCR assays. PLoS ONE 2011, 6, e21610. [CrossRef] [PubMed]

23. Hammitt, L.L.; Kazungu, S.; Welch, S.; Bett, A.; Onyango, C.O.; Gunson, R.N.; Scott, J.A.G.; Nokes, D.J. Added value of an oropharyngeal swab in detection of viruses in children hospitalized with lower respiratory tract infection. J. Clin. Microbiol. 2011, 49, 2318-2320. [CrossRef] [PubMed]

Publisher's Note: MDPI stays neutral with regard to jurisdictional claims in published maps and institutional affiliations.

(C) 2020 by the authors. Licensee MDPI, Basel, Switzerland. This article is an open access article distributed under the terms and conditions of the Creative Commons Attribution (CC BY) license (http:/ / creativecommons.org/licenses/by/4.0/). 